\title{
Commissioning of the UCLA PEGASUS Photoinjector Laboratory*
}

\author{
S. Telfer, S. Reiche, J.B. Rosenzweig, P. Frigola and G. Andonian \\ Department of Physics and Astronomy, University of California, Los Angeles
}

\section{Abstract}

The PEGASUS Photoinjector has been commissioned at UCLA. The Plane Wave Transformer Photoinjector provides a high-brightness $17 \mathrm{MeV}$ beam that will be used for numerous beam-radiation studies. Some of these will include SASE FEL, transition radiation from non-standard surfaces, and monochromatic x-ray production via PXR and Thompson scattering.

\section{INTRODUCTION}

The PEGASUS (Photoelectron Generated Amplified Spontaneous Radiation Source) laboratory has been commissioned at UCLA. First and foremost, PEGASUS is a linac-based beam-radiation laboratory. As such the injector is currently built around the Plane Wave Transformer Photoinjector (PWTP), a compact and integrated $17 \mathrm{MeV}$ photoinjector. Table 1 shows the relevant beam parameters. Currently, the PWTP has been conditioned to $20 \mathrm{MW}$ of RF power and dark-current energy has been measured to be $15 \mathrm{MeV}$ at $1 \mathrm{nC}$. We have recently put laser on the cathode and expect to characterize the photoelectron beam soon.

\begin{tabular}{ll}
\multicolumn{2}{c}{ Table 1: PEGASUS Parameters } \\
\hline Value & Parameter \\
\hline Energy & $17 \mathrm{MeV}$ \\
Energy Spread & $0.15 \%$ \\
Emittance & $1 \mathrm{~mm}-\mathrm{mrad}$ \\
Charge & $1 \mathrm{nC}$ \\
RMS Bunch Length & $1 \mathrm{~mm}$ \\
RMS Beam Size & $120 \mu \mathrm{m}$ \\
\hline
\end{tabular}

In the near term, experiments at PEGASUS will focus on beam-radiation interaction studies. The first to be completed will be the study of optical transition radiation from statistically rough, parabolic, and generalized grating surfaces. We have developed an analytical model for these processes [1]. Concurrently we will proceed with SASE FEL studies using the UCLA/KURCHATOV 2m IR FEL that was utilized in the 1998 UCLA/LANL experiment [2]. We have recently proposed to use the PEGASUS laboratory as a Thompson source for the generation of quasimonochromatic xrays up to $10 \mathrm{keV}$. This proposal is a further development of our collaboration with LLNL with its Thompson source. As a first step toward the diagnosis and transport of xray we will explore paramatric xray (PXR) production.

\footnotetext{
* Work supported by DOE grant DE-FG03-98ER45693
}

\section{DESCRIPTION OF THE LABORATORY}

\subsection{RF Photoinjector}

The PWTP is a novel standing wave S-band photocathode electron source that is designed to provide $1 \mathrm{nC} 17$ $\mathrm{MeV}$ electron beams. The PWTP consists of a $60 \mathrm{~cm}$ long, $12 \mathrm{~cm}$ diameter tank loaded with 11 discs, creating 10 full and two half accelerating cells. The peak gradients are between $40 \mathrm{MV} / \mathrm{m}$ and $60 \mathrm{MV} / \mathrm{m}$. The PWTP has a shunt impedance of approximately $50 \mathrm{M} \Omega / \mathrm{m}$ and a $Q_{L}$ of roughly 6000. This makes the PWTP an efficient structure with a moderate fill time of $2-3 \mu \mathrm{s}$. Vacuum at the cathode is maintained at less than $210^{-9}$ mbar.

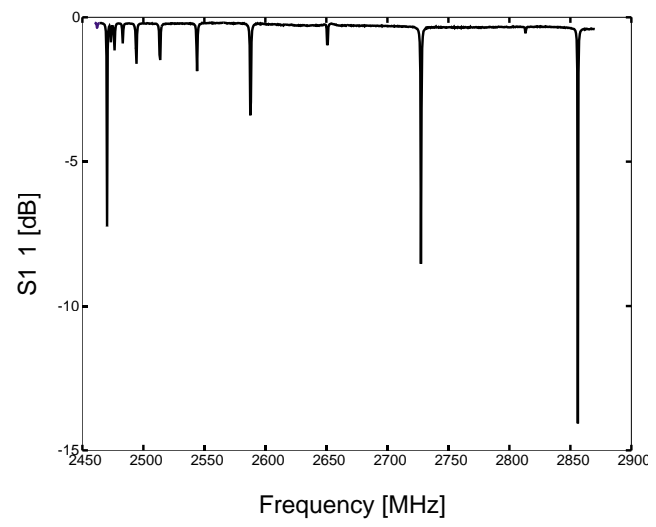

Figure 1: Modes of the PWT Photoinjector.

Fig. 1 shows the mode profile for the PWT. The $\pi$-mode is a hybrid of the TEM mode supported between the tank and discs and the $\mathrm{TM}_{01}$ supported by the disc irises. This hybridization provides excellent cell to cell coupling and a $0-\pi$ mode separation of over $400 \mathrm{MHz}$. Additionally, the RF coupling hole is symmetrized by a vacuum pump port in order to suppress RF multipole fields. The compact design allows for a simple emittance compensation solenoid. Cathode source flexibility is enhanced by a insertable cathode. Sources at PEGASUS will include OHFC copper, single crystal copper, and $\mathrm{Cs}_{2} \mathrm{Te}$.

\subsection{Photocathode Drive Laser}

The $60 \mu \mathrm{J}, 10 \mathrm{ps}$ pulses required to provide the $10 \mathrm{ps}$, $1 \mathrm{nC}$ electron beam are supplied by a ND:YLF amplifier seeded by a ND:YLF laser oscillator. The oscillator cavity is acousto-optically mode locked at $89.25 \mathrm{MHz}$, and timing is stabilized by a feedback circuit. The amplifier consists of an astigmatically compensated cavity, diode-pumped 


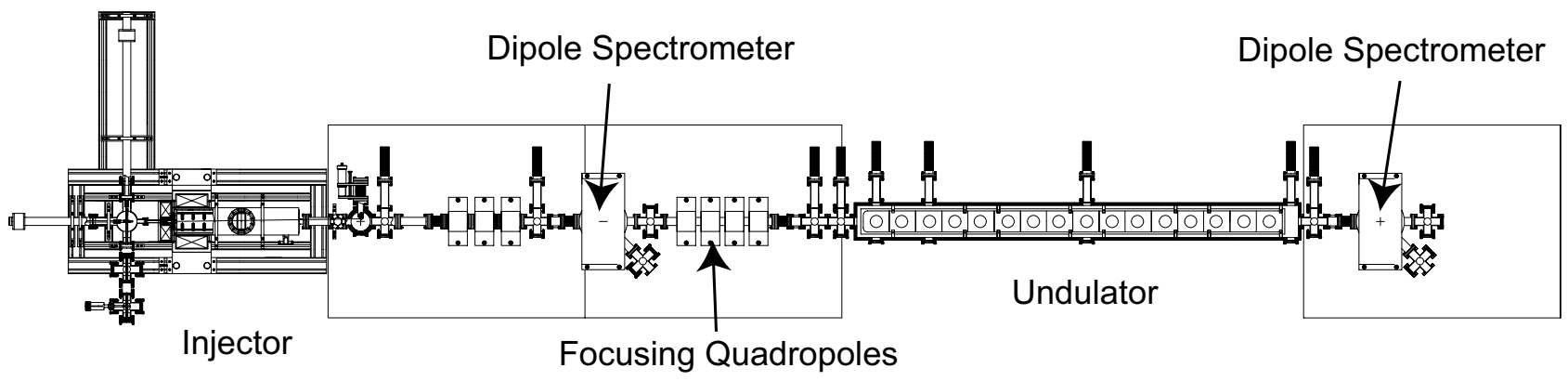

Figure 2: the PEGASUS beamline

ND:YLF rods and a quarter-wave switched Pockel's cell. The pulse repetition rate is $25 \mathrm{~Hz}$, while power output from the amplifier is $1 \mathrm{~mJ}$. Non-critical phase matching (NCPM) of a $\mathrm{LiB}_{3} \mathrm{O}_{5}$ (LBO) crystal is used for second harmonic generation, while type-I angle-tuned critical phase matching of a BetaBarium Borate (BBO) crystal is used to generate the fourth harmonic at $263 \mathrm{~nm}$. To help align the drive laser to the photocathode, the UV light is co-propagated with a HeNe laser which is imaged on the cathode.

\subsection{RF Power System}

The RF power system is designed to supply $20 \mathrm{MW}$ of power to the PWT photoinjector. A small amount of the 89.25 MHz mode-locker RF from the laser oscillator is fed into a phase locked oscillator running at $2.856 \mathrm{GHz}$. This provides a low-level RF signal which is phase-locked to the laser pulse. This CW signal is amplified by a CW amplifier to $30 \mathrm{dBm}$, and again amplified to $60 \mathrm{dBm}$ by a pulsed triode amplifier. Final amplification of the signal is made by a SLAC XK5 klystron. A transmission line transformer modulator, consisting of a twelve stage pulse forming network, $50 \mathrm{kV}$ power supply, hydrogen thyratron and SCR thyratron trigger system, provides the $4 \mu \mathrm{s}$ high voltage pulses for the XK5 klystron. The $20 \mathrm{MW}$ of RF power is transported to the PWT by a $\mathrm{SF}_{6}$ filled $\mathrm{Al}$ waveguide system. The XK5 window is protected from standing waves and large reflected voltages by a high power $\mathrm{SF}_{6}$ filled ferromagnetic isolator. Forward and reflected power are monitored by bi-directional waveguide couplers.

\subsection{Electron Beam Transport and Diagnostics}

The PEGASUS beamline is shown schematically in Fig. 2. Quadrupole magnets provide transverse focusing of the beam before and after the first spectrometer. Beam position and transverse size is monitored by intercepting the beam with $0.18 \% \mathrm{Ce}$ :YAG crystals which are imaged with CCD cameras. Beam charge is measured using impedance matched stripline BPM sum-signals and Faraday cup beam dumps. Beam energy is measured using dipole spectrometers.

When measuring the emittance of a ultra-high brightness beam like that of the PWTP, space-charge effects must be properly taken into account. A useful figure of merit is the ratio of space-charge to emittance terms taken from the rms envelope equation,

$$
R=\frac{2 I \sigma_{0}^{2}}{I_{0} \gamma \epsilon_{n}^{2}},
$$

where $I_{0}=e c / r_{e}$ is the Alfven current and $\epsilon_{n}$ is the normalized emittance. For the PWTP beam, $R>>1$ and non-linear space-charge effects clearly dominate. Measurement techniques which rely on linear transport theory, such as quad scans, will be inaccurate. In such cases it is usually preferable to use a device which separates the beam into emittance-dominated beamlets, such as slits or a pepper pot. However, a slit-based measurement of the PWTP beam would require the slit width to be less than $25 \mu \mathrm{m}$. This design requirement presents substantial practical difficulties. As such, emittance measurements at PEGASUS will be made with a quad scans. We have developed an analytical model which makes corrections to the linear transport analysis.

\section{PLANNED EXPERIMENTS}

\subsection{SASE FEL Physics}

SASE FEL studies will involve a $2 \mathrm{~m}$ planar magnet undulator. Table 2 shows relevant FEL parameters. The undulator was constructed in collaboration with the Kurchatov Institute. The undulator is enclosed in a vacuum box which maintains a pressure of less than $310^{-7}$ mbar. A pulsed wire apparatus has been developed at UCLA which was used successfully used in the UCLA/LANL experiment. The apparatus allows for multipole B-field measurement, and thereby facilitates alignment and measurement of the undulator parameter.

As mentioned above, our studies have shown that inserting a waveguide or focusing array will a significant performance enhancement by compensating for diffraction effects in the undulator. Fig. 3 shows the expected FEL performance. Saturation is clearly evident in the top curve, which shows performance with a $1 \mathrm{~mm}$ rectangular guide. The bottom curve shows the performance without any structures. Intermediate curves show performance for guides progressively larger than $1 \mathrm{~mm}$. 
Table 2: PEGASUS SASE FEL Parameters

\begin{tabular}{ll}
\hline Value & Parameter \\
\hline Matched Beam Radius & $100 \mu \mathrm{m}$ \\
Undulator Parameter & 1.05 \\
Undulator Period & $20.5 \mathrm{~mm}$ \\
Number of Periods & 98 \\
Beta Function & $22 \mathrm{~cm}$ \\
SASE Wavelength & $13.5 \mu \mathrm{m}$ \\
\hline
\end{tabular}

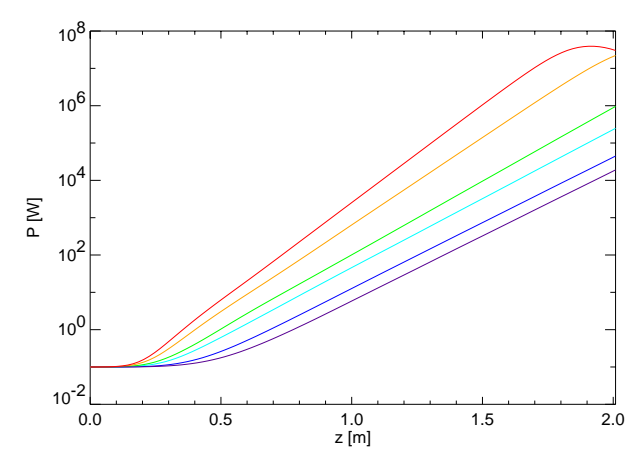

Figure 3: FEL enhancement with guide structure.

\subsection{Transition Radiation From Non-Standard Surfaces}

We have developed an extended analytical model for transition radiation from generic surfaces. Fig. 4 shows a simulated radiation pattern from a statistically rough surface intercepting a $350 \mathrm{MeV}$ electron beam. We will verify these results for $17 \mathrm{MeV}$ and also study parabolic, grating, and Fresnel surfaces, among others.

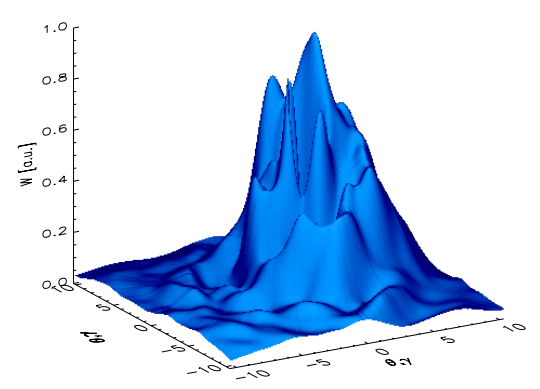

Figure 4: Simulated transition radiation from a statistically rough surface intercepting a $350 \mathrm{MeV}$ electron beam.

\subsection{Monochromatic X-Ray Production}

The PEGASUS laboratory is part of an program at UCLA to produce, diagnose and transport quasimonochromatic xray. Femtosecond pulses of quasimonochromatic $\mathrm{x}$-rays have manifold applications in medicine, materials science and chemistry. Thompson sources based on compact linear electron accelerators equipped with terawatt-class lasers are a bridge to 4th generation light sources like the Linac Coherent Light Source (LCLS). Much progress needs to be made in the diagnosis and transport of these x-ray beams. We have an ongoing collaboration with LLNL.

As such we plan to investigate $\mathrm{x}$-ray production up to the LCLS limit of approximately $12 \mathrm{keV}$.

As initial step, we plan to generate Parametric xray (PXR) with the PWTP electron beam. PXR is generated by the effective Bragg diffraction of virtual photons associated with the passage of a highly-relativistic electron beam through a crystal [5]. Tunability is possible by rotating the crystal orientation. Photon/electron yields for pyrolytic graphite of $10^{-6}$ are common in the literature [6].

UCLA has recently proposed a Thompson source based on the PWTP beam and a table-top terawatt laser, which would provide $\mathrm{x}$-rays up to $12 \mathrm{keV}$ and and peak brightness on the order of $10^{-2} \mathrm{x}$-rays/electron. It has been proposed that this source could function as a user facility for the California Nanosystems Institute (CNSI), which is currently being built at UCLA.

\section{REFERENCES}

[1] S. Reiche, J. Rosenzweig, Presented at this Conference.

[2] M. Hogan, et. al., Physical Review Letters, 8122 (1998) 4867.

[3] S. Reiche, et. al. Proc. of the 2000 Free Electron Laser Conference, Duke 2000.

[4] LCLS Design Study report, Report SLAC-R-521 (1998).

[5] e.g. S.A. Vorob'ev et al., Sov. phys. JETP Lett.41,1 (1985); V.G. Baryshevsky et al. Phys. Lett. 110A, 477 (1985); Yu. N. Adishev et al. Nucl. Inst. and Methods A248,28 (1987); A.V. Shchagin et al. Phys Lett. A148, 458 (1990); V.P. Afanesenko et al., Phys Lett. A141, 313 (1989).

[6] See [5] and e.g. X.K. Maruyama et. al., Proc. of the 1993 Particle Accelerator Conference, p.1620-2 vol.2. 